\title{
An Efficient Algorithm for Space-Time Block Code Classification
}

\author{
Yahia A. Eldemerdash, Octavia A. Dobre, Mohamed Marey ${ }^{\dagger}$, George K. Karagiannidis ${ }^{\ddagger}$, and Bruce Liao ${ }^{\dagger \dagger}$ \\ Faculty of Engineering and Applied Science, Memorial University of Newfoundland, St. John's, NL, Canada \\ $\dagger$ Faculty of Electronic Engineering, Menoufyia University, Menouf, Egypt \\ ${ }^{\ddagger}$ Department of Electrical and Computer Engineering, Aristotle University of Thessaloniki, Greece \\ ${ }^{\dagger \dagger}$ Defence Research and Development Canada, Ottawa, ON, Canada \\ Email: \{yahia.eldemerdash, odobre\}@mun.ca, ${ }^{\dagger}$ mfmmarey@mun.ca, ${ }^{\ddagger}$ geokarag@auth.gr, \\ $\dagger^{\dagger}$ bruce.liao@drdc-rddc.gc.ca
}

\begin{abstract}
This paper proposes a novel and efficient algorithm for space-time block code (STBC) classification, when a single antenna is employed at the receiver. The algorithm exploits the discriminating features provided by the discrete Fourier transform (DFT) of the fourth-order lag products (FOLPs) of the received signal. It does not require estimation of the channel, signal-to-noise ratio (SNR), and modulation of the transmitted signal. Computer simulations are conducted to evaluate the performance of the proposed algorithm. The results show the validity of the algorithm, its robustness to carrier frequency offset, and low sensitivity to timing offset.
\end{abstract}

Index Terms-Signal classification, space-time block code (STBC).

\section{INTRODUCTION}

Blind classification of communication signals plays a pivotal role in both civilian and military applications, e.g., parameter configuration in software-defined radio, spectrum awareness in cognitive radio, and spectrum monitoring and surveillance [1]-[3].

A large number of studies have been carried out for developing blind signal classification algorithms in singleinput single-output scenarios (see the comprehensive survey [3] and references therein). Regarding multiple-input multipleoutput (MIMO) technology, which has been recently included in wireless standards such as IEEE 802.11n, IEEE 802.16e, and 3GPP LTE [4], new and challenging signal classification problems have arisen. These relate to the estimation of the number of transmit antennas, as well as space-time code and modulation classification. The research on signal classification for MIMO scenarios is at an incipient stage. Blind estimation of the number of transmit antennas is investigated in [5], [6], and modulation classification for spatial multiplexing (SM) in [7]-[10], while blind classification of linear spacetime block codes (STBCs) has been recently explored in [11]-[17]. Classification algorithms can be mainly divided into two categories: likelihood-based [11] and feature-based algorithms [12]-[17]. Likelihood-based algorithms calculate the likelihood function of the received signal, and employ the maximum likelihood criterion for decision making [11]. However, these algorithms require channel estimation, time, block, and frequency synchronization, and knowledge of the modulation format. Furthermore, they suffer from high com- putational complexity. Second-order statistics are exploited in [12], [13], while fourth-order statistics are considered in [14]. In [15]-[17], signal cyclostationarity-based features are used. Most of these investigations assume perfect timing and frequency synchronization [11]-[16]. Some of these papers study the classification of SM and Alamouti (AL)-STBC [14][16], whereas others consider a larger pool of STBCs [11][13], [17]. Furthermore, most employ multiple antennas at the receiver [12], [13], [16], [17]. Since this requirement can not always be met, solutions for STBC blind classification when a single receive antenna is available are of interest.

This paper proposes an efficient algorithm for the linear STBC classification, which exploits features based on the discrete Fourier transform (DFT) of the fourth-order lag products (FOLPs) of the received signal. The proposed algorithm does not require information about channel, modulation, and signalto-noise ratio (SNR). Moreover, it is robust to carrier frequency offset and requires only a rough estimate of clock timing.

The rest of this paper is organized as follows: Section II introduces the signal model. Section III describes the proposed STBC classification algorithm. Simulation results are presented in Section IV. Finally, concluding remarks are drawn in Section V.

\section{SignAL MODEL}

We consider a wireless communication system which employs linear STBCs with multiple transmit antennas. In such a case, a block of $N_{s}$ modulated symbols is encoded to generate $N_{t}$ parallel signal sequences of length $L$, to be transmitted simultaneously with $N_{t}$ antennas in $L$ consecutive time periods [18]. We denote the $b$ th block of $N_{s}$ complex symbols to be transmitted by the column vector $\mathbf{X}_{b}=\left[x_{b, 0}, \ldots, x_{b, N_{s}-1}\right]^{T}$, with the superscript $T$ denoting transposition. The symbols are zero-mean independent and identically distributed (i.i.d) random variables, with values drawn from an alphabet corresponding to an $M$-PSK or $M$-QAM, $M \geq 4$, signal constellation. Without loss of generality, we consider unit variance constellations, i.e., $\mathrm{E}\left\{|x|^{2}\right\}=1$, with $\mathrm{E}\{$.$\} as the statistical$ expectation. Further, we denote the $N_{t} \times L$ space-time coding matrix and the $(l+1)$ th column of this matrix by $\mathbb{G}\left(\mathbf{X}_{b}\right)$ and $\mathbb{G}_{l}\left(\mathbf{X}_{b}\right), 0 \leq l<L$, respectively. 
The received signal is assumed to be encoded by one of the following STBCs ${ }^{1}$ : SM [18] with $N_{t}=2$ and $L=1$, AL code [18] with $N_{t}=2$ and $L=2$ (orthogonal with rate 1), STBC3 [18] with $N_{t}=3$ and $L=4$ (orthogonal with rate $\frac{3}{4}$ ), or STBC4 [19] with $N_{t}=3$ and $L=8$ (orthogonal with rate $\frac{1}{2}$ ).

For example, the transpose coding matrix of STBC4 is defined as [19]

$$
\left(\mathbb{G}^{\mathrm{STBC} 4}\left(\mathbf{X}_{b}\right)\right)^{T}=\left[\begin{array}{ccc}
x_{b, 0} & x_{b, 1} & x_{b, 2} \\
-x_{b, 1} & x_{b, 0} & -x_{b, 3} \\
-x_{b, 2} & x_{b, 3} & x_{b, 0} \\
-x_{b, 3} & -x_{b, 2} & x_{b, 1} \\
x_{b, 0}^{*} & x_{b, 1}^{*} & x_{b, 2}^{*} \\
-x_{b, 1}^{*} & x_{b, 0}^{*} & -x_{b, 3}^{*} \\
-x_{b, 2}^{*} & x_{b, 3}^{*} & x_{b, 0}^{*} \\
-x_{b, 3}^{*} & -x_{b, 2}^{*} & x_{b, 1}^{*}
\end{array}\right],
$$

where the rows and columns correspond to the time periods and transmit antennas, respectively.

We consider a receiver equipped with a single antenna, and assume that the STBC time alignment and length are unknown, whereas symbol and carrier synchronization are performed. Later in the paper, we will study the effect of timing and carrier frequency offsets. Without loss of generality, we assume that the first received symbol, denoted by $r(0)$, intercepts the $\left(k_{1}+1\right)$ th column, $0 \leq k_{1}<L$, of the $b$ th transmitted block, denoted by $\mathbb{G}_{k_{1}}\left(\mathbf{X}_{b}\right)$. Under these assumptions, the $k$ th received symbol, $r(k), k \geq 0$, is expressed as [12]

$$
r(k)=\mathbf{H S}(k)+w(k),
$$

where $\mathbf{S}(k)=\mathbb{G}_{p}\left(\mathbf{X}_{q}\right)$, with $p=\left(k+k_{1}\right) \bmod L, q=$ $b+\left(k+k_{1}\right) \operatorname{div} L$, and $z \bmod L$ and $z \operatorname{div} L$ denoting respectively the remainder and the quotient of the division $z / L, \mathbf{H}=\left[h_{0}, \ldots, h_{N_{t}-1}\right]$ represents the vector of the channel coefficients, which characterize the paths between the transmit and receive ends, and $w(k)$ represents the complex additive white Gaussian noise (AWGN) with zero mean and variance $\sigma_{w}^{2}$.

\section{AN EFFICIENT CLASSIFICATION ALGORITHM}

In this section, we show how the DFT of the FOLP of the received signal can be exploited to blindly classify the STBCs under consideration based on $K$ received symbols, $r(k), 0 \leq$ $k \leq K-1$, when a single receive antenna is employed.

\section{A. Discriminating features}

The FOLP of the received sequence, $\{r(k)\}_{k=0}^{K-1}$, at delay vector $[0,0, \tau, \tau]$ is defined as

$$
y(k, \tau)=r^{2}(k) r^{2}(k+\tau), \quad k=0,1, \ldots, K-1 .
$$

\footnotetext{
${ }^{1}$ We choose AL and SM as they are the most commonly used in wireless standards [4], and STBC3 and STBC4, as being commonly referred codes [18], [19]
}

Based on the structure of the code matrices, we first consider $\tau=4$ due to its discriminating property for STBC4, as it is subsequently explained.

Without loss of generality, we assume that the first received symbol, $r(0)$, corresponds to the start of the STBC block. Later we will show that this assumption will not affect the performance of the proposed algorithm.

By substituting (2) in (3) of the FOLP, one can express the FOLPs corresponding to the considered STBCs as

$$
y^{\operatorname{STBC}(k, 4)}= \begin{cases}a(k)+\psi_{1}(k) & \text { under event } \Im_{1} \\ \psi_{2}(k) & \text { under event } \Im_{2}\end{cases}
$$

and

$$
y^{\xi}(k, 4)=\psi_{3}^{\xi}(k), \quad \xi \in\{\mathrm{SM}, \mathrm{AL}, \mathrm{STBC} 3\},
$$

where $\Im_{1}$ is the event that $r(k)$ and $r(k+4)$ belong to the same transmitted data block, $\Im_{2}$ is the event that they do not, and $a(k)=[\mathbf{H S}(k)]^{2}[\mathbf{H S}(k+4)]^{2}$. Furthermore, $\psi_{1}(k)$, $\psi_{2}(k)$, and $\psi_{3}(k)$ represent the remaining signal and AWGN components.

Note that $a(k)$ depends on the channel coefficients and the transmitted data symbols corresponding to $r(k)$ and $r(k+4)$ under event $\Im_{1}$. For example, one can show that $a(k=b L)$ is given by

$$
\begin{aligned}
a(b L)= & 4\left(\left|x_{b, 0}\right|^{2}\left|x_{b, 1}\right|^{2} h_{0}^{2} h_{1}^{2}+\left|x_{b, 0}\right|^{2}\left|x_{b, 2}\right|^{2} h_{0}^{2} h_{2}^{2}\right. \\
& \left.+\left|x_{b, 1}\right|^{2}\left|x_{b, 2}\right|^{2} h_{1}^{2} h_{2}^{2}\right)+\left|x_{b, 0}\right|^{4} h_{0}^{4} \\
& +\left|x_{b, 1}\right|^{4} h_{1}^{4}+\left|x_{b, 2}\right|^{4} h_{2}^{4},
\end{aligned}
$$

where $x_{b, 0}, x_{b, 1}$, and $x_{b, 2}$ are the data symbols transmitted in the first time period of the $b$ th STBC4 block. Similar expressions can be also obtained for $a(k), k \neq b L$. It is noteworthy that $a(k)$ is constant for the $M$-PSK signals, i.e., it does not depend on the transmitted symbols, as PSK is a constant envelope modulation. For $M$-QAM signals, $a(k)$ can be expressed as a constant, $A_{\mathrm{QAM}}=\mathrm{E}\{a(k)\}$, plus a variable $\psi_{4}(k)$, which represents the deviation from the mean ${ }^{2}$. For the convenience of notation, subsequently we use the constant $A \in\left\{A_{\mathrm{PSK}}, A_{\mathrm{QAM}}\right\}$, which is given by

$A=m_{x, 4,2}\left(h_{0}^{4}+h_{1}^{4}+h_{2}^{4}\right)+4\left(m_{x, 2,1}\right)^{2}\left(h_{0}^{2} h_{1}^{2}+h_{0}^{2} h_{2}^{2}+h_{1}^{2} h_{2}^{2}\right)$,

with $m_{x, \alpha, \beta}=\mathrm{E}\left\{x^{\alpha-\beta}\left(x^{*}\right)^{\beta}\right\}$ as the $(\alpha, \beta)$ moment of the points in the signal constellation. Examples of $m_{x, 4,2}$ values are provided in Table I for various unit variance signal constellations [3].

In the absence of the noisy components, i.e., $\psi_{1}(k)+$ $\psi_{4}(k)=\psi_{2}(k)=\psi_{3}(k)=0$, the FOLP sequence is

- STBC4: [ A A A A 00000 A A A A 000000 ...] and

- STBC3, AL, and SM: $\left[\begin{array}{llll}0 & 0 & 0 & \ldots . .\end{array}\right]$.

${ }^{2}$ Generally speaking, a random variable $x$ can be expressed as $x=\mathrm{E}\{x\}+$ $\nu$, where $\nu$ is a zero-mean random variable which represents the deviation from the mean $\mathrm{E}\{x\}[20]$. 
TABLE I

MOMENT VALUES FOR VARIOUS SIGNAL CONSTELLATIONS.

\begin{tabular}{|c|c|c|c|c|}
\hline & QPSK & 8-PSK & 16-QAM & 64-QAM \\
\hline \hline$m_{x, 2,1}$ & 1 & 1 & 1 & 1 \\
\hline$m_{x, 4,2}$ & 1 & 1 & 1.32 & 1.38 \\
\hline
\end{tabular}

Thus STBC4 can be recognized by detecting the periodicity of its FOLP at $\tau=4$, for which we employ the DFT. Let $\mathbf{Y}=[Y(0, \tau), Y(1, \tau), \ldots, Y(K-1, \tau)]$ denote the $K$-point DFT of $\mathbf{y}$, with

$Y(n, \tau)=\frac{1}{\sqrt{K}} \sum_{k=0}^{K-1} y(k, \tau) e^{-j 2 \pi k n / K}, \quad n=0,1, \ldots, K-1$.

By replacing (4) and (5) in (8), it follows that

$Y^{\mathrm{STBC} 4}(n, 4)= \begin{cases}\mathcal{A}(n)+\Psi_{1}(n) & n=0, \frac{K}{8}, \frac{3 K}{8}, \frac{5 K}{8}, \frac{7 K}{8}, \\ \Psi_{1}(n) & \text { otherwise, }\end{cases}$

and

$$
Y^{\xi}(n, 4)=\Psi_{2}(n), \quad \xi \in\{\mathrm{SM}, \mathrm{AL}, \mathrm{STBC} 3\},
$$

where $\Psi_{1}(n)$ and $\Psi_{2}(n)$ represent the DFT of the noisy components, and $\mathcal{A}(n)$ represents the peak values at $n=0, \frac{K}{8}, \frac{3 K}{8}, \frac{5 K}{8}, \frac{7 K}{8}$, which depend on $A$ and $K$. One can easily show that $\mathcal{A}(0)=\frac{\sqrt{K}}{2} A, \mathcal{A}\left(\frac{K}{8}\right)=$ $\mathcal{A}\left(\frac{7 K}{8}\right)=\frac{\sqrt{K}}{8} A(1-i(1+\sqrt{2}))$, and $\mathcal{A}\left(\frac{3 K}{8}\right)=\mathcal{A}\left(\frac{5 K}{8}\right)=$ $\frac{\sqrt{K}}{8} A(1+i(1-\sqrt{2}))$.

The inspection of (9) and (10) indicates that $|Y(n, 4)|$ exhibits 5 peaks at $n=0, \frac{K}{8}, \frac{3 K}{8}, \frac{5 K}{8}, \frac{7 K}{8}$ for STBC4, while it does not for the other codes; thus, this discriminating feature can be exploited to recognize $\operatorname{STBC}^{3} .\left|Y^{\mathrm{STBC}_{4}}(n, 4)\right|$ is shown as a function of $n$ in Fig. 1. Based on the vector $Y(n, 4)$, we define the vector $Z(u, 4)$,

$$
Z(u, 4)=\sum_{l=0}^{3}\left|Y\left(\frac{l K}{4}+u, 4\right)\right|^{2}, \quad u=0,1, \ldots, \frac{K}{4}-1 .
$$

For STBC4, $Z^{\operatorname{STBC} 4}(u, 4)$ exhibits two peaks at $u=0, \frac{K}{8}$, where

$$
Z^{\mathrm{STBC} 4}(0,4)=\left|Y^{\mathrm{STBC} 4}(0,4)\right|^{2},
$$

and

$$
\begin{aligned}
Z^{\operatorname{STBC} 4}\left(\frac{K}{8}, 4\right) & =\left|Y^{\operatorname{STBC} 4}\left(\frac{K}{8}, 4\right)\right|^{2}+\left|Y^{\operatorname{STBC} 4}\left(\frac{3 K}{8}, 4\right)\right|^{2} \\
& +\left|Y^{\operatorname{STBC} 4}\left(\frac{5 K}{8}, 4\right)\right|^{2}+\left|Y^{\operatorname{STBC}}\left(\frac{7 K}{8}, 4\right)\right|^{2}
\end{aligned}
$$

Note that this operation reduces the number of peaks to two for STBC4, while it strengthens the second peak value.

${ }^{3}$ Note that timing misalignment will introduce a cyclic shift in the FOLP sequence [A A A A 0000 A A A A ...], which will affect neither the positions nor the magnitudes of the DFT peaks.

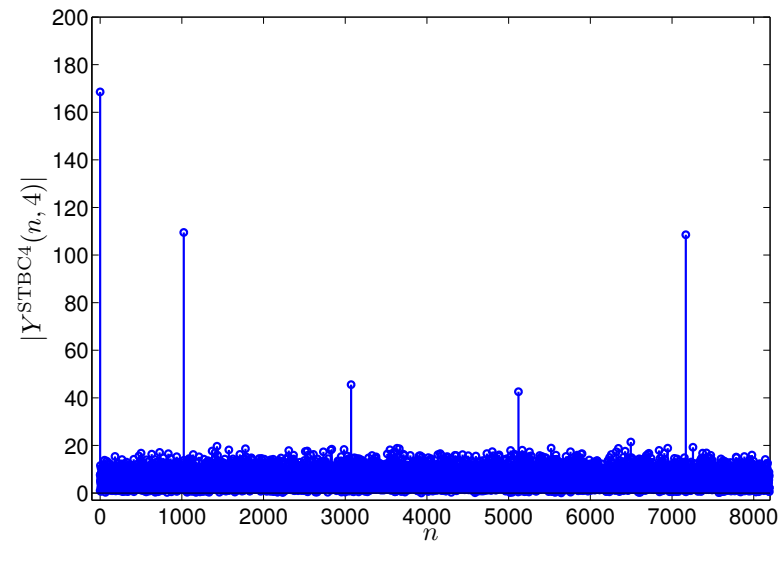

Fig. 1. $\left|Y^{\operatorname{STBC}_{4}}(n, 4)\right|$ with QPSK modulation and $K=8192$, at SNR=20 dB over Nakagami- $m$ fading channel, $m=3$.

Furthermore, we should note that $Z(u, 4)$ has no peaks for STBC3, AL, and SM.

Similarly, one can show that for $\tau=1$ and in the absence of the noisy components, the FOLP sequence is

- STBC3: [0 B1 B2 00 B1 B2 0 ...], with

$$
B_{1}=2\left(m_{x, 4,2}-2\left(m_{x, 2,1}\right)^{2}\right) h_{1}^{2} h_{2}^{2},
$$

and

$$
B_{2}=2\left(m_{x, 4,2}-2\left(m_{x, 2,1}\right)^{2}\right) h_{0}^{2} h_{1}^{2} .
$$

- AL: [C 0 C 0 C $0 . .$.$] , with$

$$
C=2\left(m_{x, 4,2}-2\left(m_{x, 2,1}\right)^{2}\right) h_{0}^{2} h_{1}^{2} .
$$

- SM: $\left[\begin{array}{llll}0 & 0 & 0 & \ldots\end{array}\right]$.

In this case, $|Y(n, 1)|$ exhibits four peaks at $n=0, \frac{K}{4}$, $\frac{K}{2}, \frac{3 K}{4}$ for STBC3, and two peaks at $n=0, \frac{K}{2}$ for AL.

We further define the vector $Z(u, 1)$ as,

$Z(u, 1)=\sum_{l=0}^{1}\left|Y\left(\frac{l K}{2}+u, 1\right)\right|^{2}, \quad u=0,1, \ldots, \frac{K}{2}-1$.

One can show that $Z(u, 1)$ has two peaks at $u=0, \frac{K}{4}$ for STBC3, where

$$
Z^{\mathrm{STBC} 3}(0,1)=\left|Y^{\mathrm{STBC} 3}(0,1)\right|^{2}+\left|Y^{\mathrm{STBC} 3}\left(\frac{K}{2}, 1\right)\right|^{2},
$$

and

$$
Z^{\mathrm{STBC} 3}\left(\frac{K}{4}, 1\right)=\left|Y^{\mathrm{STBC} 3}\left(\frac{K}{4}, 1\right)\right|^{2}+\left|Y^{\mathrm{STBC} 3}\left(\frac{3 K}{4}, 1\right)\right|^{2} .
$$

Note that this operation reduces the number of peaks to two for STBC3, and strengthens the peak values. Furthermore, it can be easily shown that $Z(u, 1)$ has only one peak at $u=0$ for $\mathrm{AL}$, while it has no peaks for SM.

\section{B. Decision tree algorithm for STBC classification}

STBC classification is formulated as a hypothesis testing problem, i.e., under hypothesis $\mathcal{H}_{\xi}$, the STBC $\xi \in$ $\{\mathrm{SM}, \mathrm{AL}, \mathrm{STBC} 3, \mathrm{STBC} 4\}$ is selected. The discriminating 


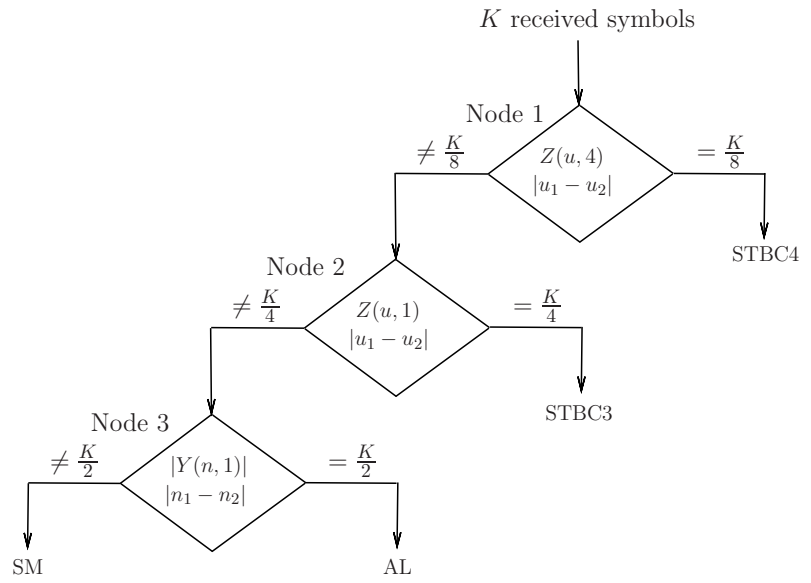

Fig. 2. Decision tree algorithm for STBC classification.

features previously described are used with a decision tree classification algorithm, which is presented in Fig. 2.

At node $1, Z(u, 4)$ is used to discriminate between STBC4 and $\{\mathrm{SM}, \mathrm{AL}, \mathrm{STBC}\}\}$ based on the existence of the two peaks in $Z(u, 4)$, whose difference in location is $\frac{K}{8}$,

$$
\left|u_{1}-u_{2}\right|=\frac{K}{8},
$$

where

$$
u_{1}=\arg \max _{u} Z(u, 4),
$$

and

$$
u_{2}=\arg \max _{u, u \neq u_{1}} Z(u, 4) .
$$

At node 2, $Z(u, 1)$ is used to discriminate between STBC3 and $\{\mathrm{SM}, \mathrm{AL}\}$ based on the existence of the two peaks in $Z(u, 1)$, whose difference in location is $\frac{K}{4}$. In other words, if $u_{1}$ and $u_{2}$ are the positions of the two maximum values in $Z(u, 1), \mathrm{STBC} 3$ is declared present if

$$
\left|u_{1}-u_{2}\right|=\frac{K}{4} .
$$

Finally, at node 3, discrimination between AL and SM is performed based on the peak positions in $|Y(n, 1)|$ [14]. If $n_{1}$ and $n_{2}$ are the positions of the maximum values in $|Y(n, 1)|$, $\mathrm{AL}$ is recognized if

$$
\left|n_{1}-n_{2}\right|=\frac{K}{2} .
$$

Otherwise, SM is declared present.

\section{Simulation RESUltS}

\section{Simulation setup:}

Unless otherwise mentioned, the number of observed samples, $K$, was set to 8192 and quadrature phase-shift-keying (QPSK) modulation was used. The average probability of correct classification was employed as a performance measure, i.e, $P_{c}=4^{-1} \sum_{\xi \in\{\mathrm{SM}, \mathrm{AL}, \mathrm{STBC} 3, \mathrm{STBC} 4\}} P(\xi \mid \xi) . P(\xi \mid \xi)$ was estimated from 1000 Monte Carlo trials for each $\xi \in$ $\{\mathrm{SM}, \mathrm{AL}, \mathrm{STBC} 3, \mathrm{STBC} 4\}$. The received signal was affected by a frequency-flat Nakagami- $m$ fading channel [21] with

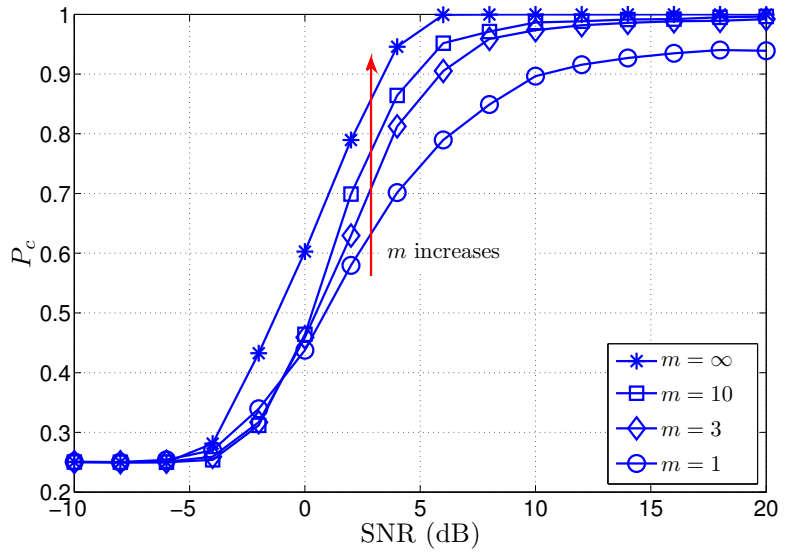

Fig. 3. Average probability of correct classification, $P_{c}$, versus SNR with QPSK modulation and $K=8192$ for diverse Nakagami- $m$ fading channels.

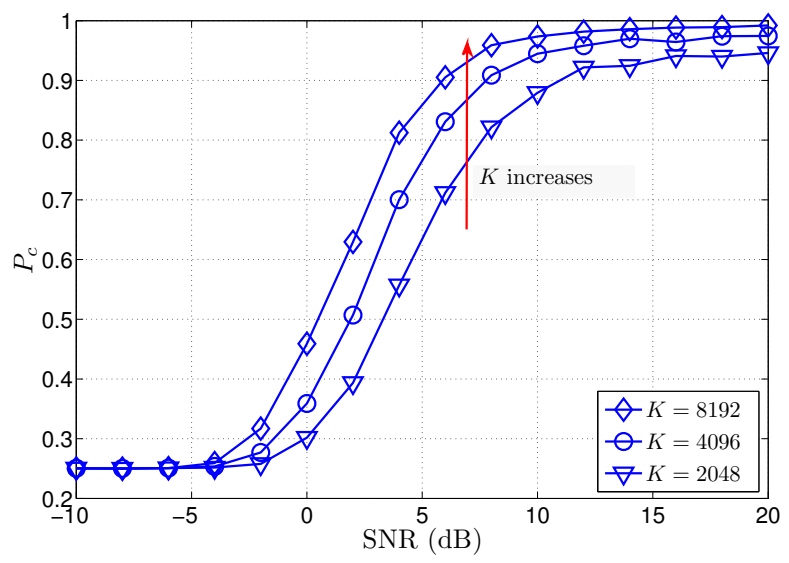

Fig. 4. Effect of the number of received samples, $K$, on $P_{c}$ with QPSK modulation over Nakagami- $m$ fading channel, $m=3$.

$m=3$, unless otherwise mentioned. Furthermore, AWGN with variance $\sigma_{w}^{2}$ was considered. Given a unit variance constellation, the SNR is defined as $10 \log _{10}\left(N_{t} / \sigma_{w}^{2}\right)$.

Performance evaluation:

Fig. 3 shows the $P_{c}$ achieved with the proposed algorithm over Nakagami- $m$ fading channel with $m=1,3,10$ and $\infty$. As expected, the performance improves as $m$ increases. For example, at $\mathrm{SNR}=10 \mathrm{~dB}, P_{c}=0.9,0.97$, and 0.986 for $m=$ 1,3 , and 10 , respectively, while it reaches 1 for $m=\infty$. This can be easily explained, as the variance of the channel coefficients increases for lower $m$ values, which affects the value of the discriminating peaks, thus, leading to erroneous decisions.

Effect of the number of observed samples:

Fig. 4 illustrates the effect of the number of received samples on the $P_{c}$. The performance enhances by increasing $K$, as this results in an increase of the peak values and a reduction of the effect of the noisy components. Note that, as the proposed algorithm depends on fourth-order statistics, a large number of observed samples is required for accurate estimation of the discriminating feature. 


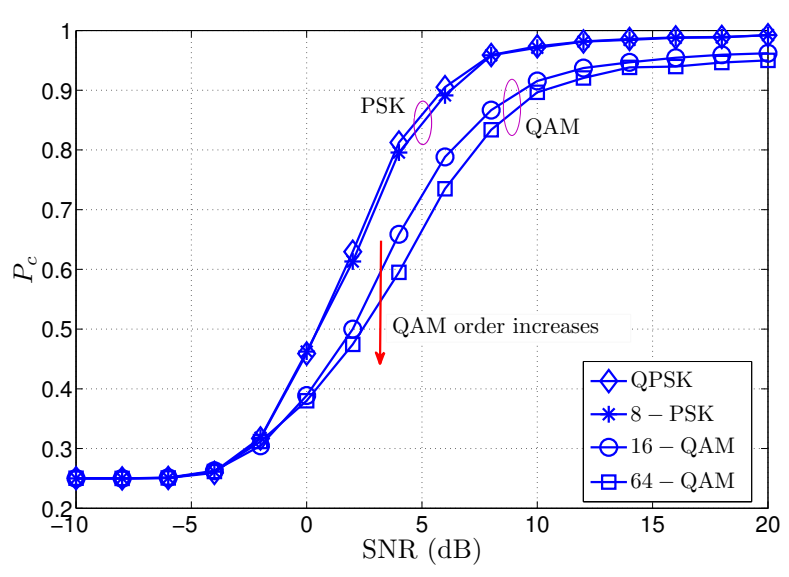

Fig. 5. Effect of the modulation type on $P_{c}$ with $K=8192$ over Nakagami$m$ fading channel, $m=3$.

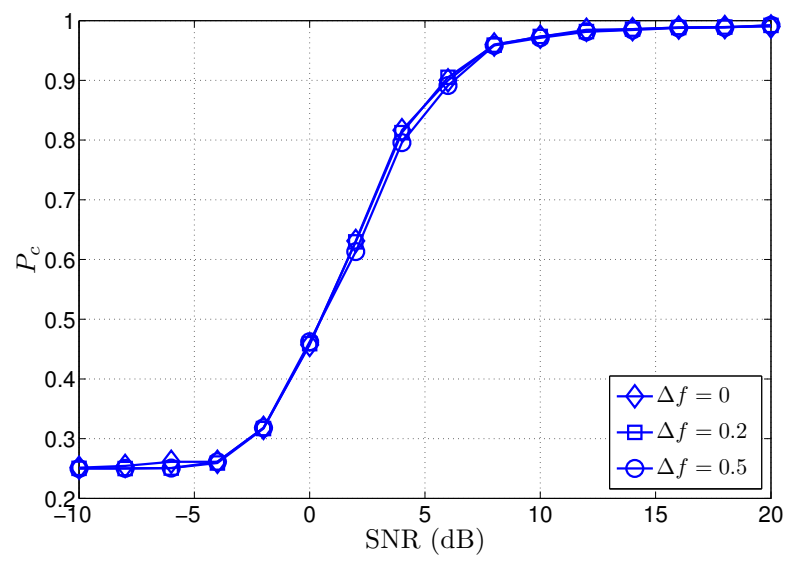

Fig. 6. Effect of the frequency offset on $P_{c}$ with QPSK modulation and $K=8192$ over Nakagami- $m$ fading channel, $m=3$.

\section{Effect of modulation type:}

Fig. 5 shows the effect of the modulation type on the $P_{c}$; a better performance is achieved for $M$-PSK signals when compared with $M$-QAM signals. The explanation is that $a(k)$ is constant for $M$-PSK, whereas it is not for $M$-QAM. This yields an increase in the variance of the noisy components as $M$ increases, leading to performance degradation.

\section{Effect of frequency offset:}

The previous analysis assumed carrier frequency synchronization. Here we evaluate the performance of the proposed algorithm in the presence of a carrier frequency offset. Fig. 6 presents the effect of the frequency offset normalized to the data rate, $\Delta f$, on the $P_{c}$. It can be noticed that the performance does not depend on $\Delta f$. This is because $\Delta f$ introduces a shift in the position of the peaks, and thus, it has no effect on the decision made on the difference between these positions. Hence, the algorithm is robust to carrier frequency offset.

\section{Effect of timing offset:}

The previous analysis additionally assumed perfect timing synchronization. Here we show the performance of the pro-

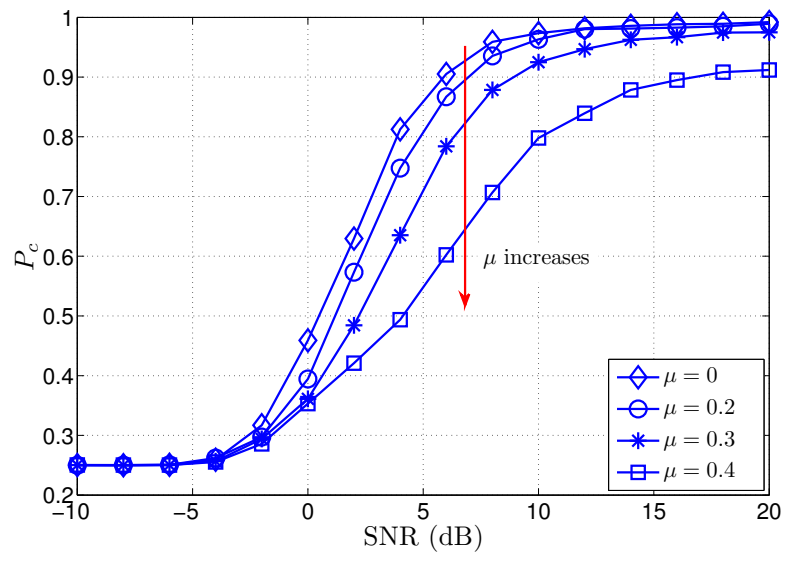

Fig. 7. Effect of the timing offset on $P_{c}$ with QPSK modulation and $K=$ 8192 over Nakagami- $m$ fading channel, $m=3$.

posed algorithm in the presence of a timing offset normalized to the sampling period, $0 \leq \mu<1$. For the case of rectangular pulse shaping, after the matched filtering, the timing offset $\mu$ translates into a two path channel $[1-\mu, \mu]$ [12]. Fig. 7 shows the $P_{c}$ for $\mu=0,0.2,0.3$ and 0.4 . The performance slightly decreases for $\mu=0.2$ and 0.3 , while it reduces more for $\mu=0.4$.

\section{Effect of the spatially correlated fading:}

Independent fading was considered in the previous analysis. Here we evaluate the classification performance under spatial correlation fading environment. Spatially correlated Nakagami- $m$ fading with correlation coefficient $\rho, 0 \leq \rho \leq 1$, is generated using correlated complex-valued Gaussian variables ([22], p. 25), the inverse cumulative distribution function (cdf) method [21], and an approximation of the Nakagami- $m$ inverse cdf [21]. Fig. 8 shows the effect of the spatial correlation between transmitted antennas on the $P_{c}$ for $\rho=0,0.5$, and 1. Note that a similar performance is obtained for $\rho=0$ and 0.5 , while the performance slightly improves for $\rho=1$. This is because the performance is basically controlled by the absolute values of the DFT peaks, which are proportional to $\left(\left|h_{i}\right|^{2}\left|h_{j}\right|^{2}\right), i, j=0,1,2, i \neq j$, and $\mathrm{E}\left\{\left|h_{i}\right|^{2}\left|h_{j}\right|^{2}\right\}$ is a slowly increasing function of $\rho$ [14].

\section{Performance comparison:}

Fig. 9 compares the $P_{c}$ obtained with the proposed algorithm, the optimal likelihood-based algorithm in [11], and the second-order correlation-based algorithm in [12]. Note that the former requires estimation of the channel coefficients, noise power, and modulation type, and results are shown for perfect estimates of these parameters. As expected, under such a scenario, it provides the best performance. On the other hand, the proposed algorithm has no such requirements, which makes it suitable for practical applications. Moreover, the computational cost measured by the required number of floating point operations (flops) [23] for the proposed algorithm is $47 K+10 K \log _{2} K$, which is significantly lower than $K\left(240 M^{2}+640 M^{3}+1652 M^{4}\right)$ for the likelihoodbased algorithm. For example, with QPSK modulation and 


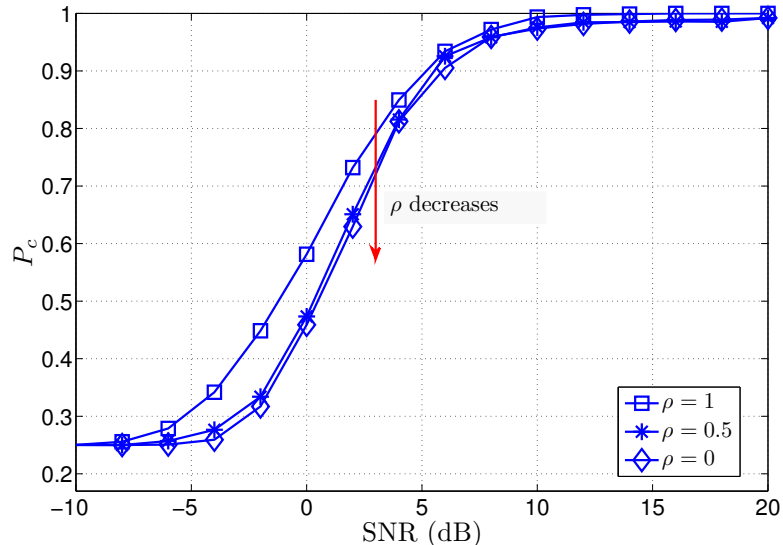

Fig. 8. Effect of the spatial correlation between transmitted antennas on $P_{c}$ with QPSK modulation and $K=8192$ over Nakagami- $m$ fading channel, $m=3$.

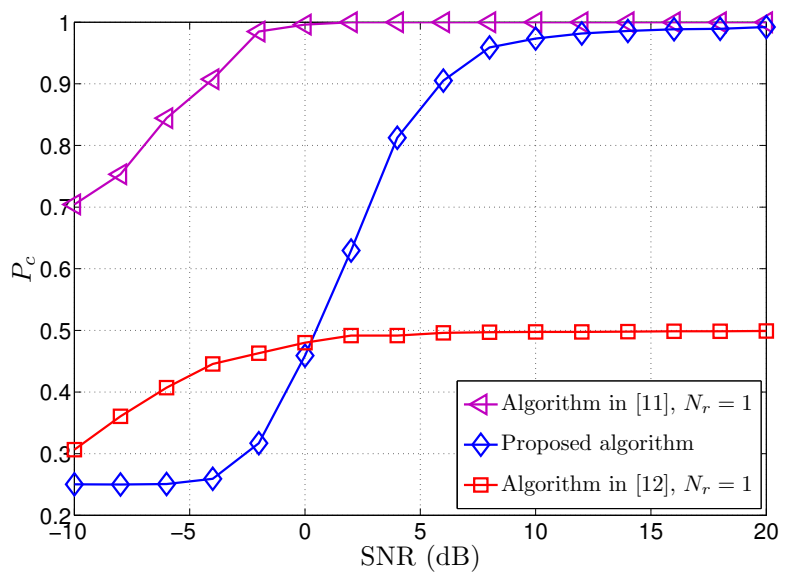

Fig. 9. Performance comparison of the proposed algorithm, the optimal likelihood-based algorithm in [11], and the second-order correlation-based algorithm in [12] with QPSK modulation and $K=8192$ over Nakagami- $m$ fading channel, $m=3$.

$K=8192$, the proposed algorithm needs only $1.45 \times 10^{6}$ flops, whereas the likelihood-based algorithm requires $3.83 \times 10^{9}$ flops. Furthermore, the proposed algorithm greatly outperforms the algorithm in [12], which achieves a $P_{c}=0.5$ even for high SNR. This can be explained as the secondorder correlation provides a discriminating feature for SM and STBC4 only. For AL and STBC3, it equals zero, leading to the mis-classification of these codes.

\section{CONCLUSION}

This paper proposed an algorithm for blind classification of space-time block codes (STBCs) using a single receive antenna. It was shown that the discrete Fourier transform (DFT) of the fourth-order lag products (FOLPs) of the received signal provides a discriminating signal feature; a decision tree classification algorithm was developed based on this feature. The algorithm performance was evaluated through simulations in terms of average probability of correct classification. The results indicated the validity of the algorithm, with the advantages that it does not require channel estimation, is robust to carrier frequency offset, and exhibits a low sensitivity to timing offset. Moreover, it can benefit from spatially correlated fading.

\section{ACKNOWLEDGEMENT}

This work has been supported in part by Defence Research and Development Canada.

\section{REFERENCES}

[1] J. L. Xu, W. Su, and M. C. Zhou, "Software-defined radio equipped with rapid modulation recognition," IEEE Trans. Veh. Technol., vol. 59, pp. 1659-1667, May 2010.

[2] T. Yucek and H. Arslan, "A survey of spectrum sensing algorithms for cognitive radio applications," IEEE Commun. Surveys Tuts., vol. 11, pp. 116-130, Mar. 2009.

[3] O. A. Dobre, A. Abdi, Y. Bar-Ness, and W. Su, "A survey of automatic modulation classification techniques: classical approaches and new developments," IET Commun., vol. 1, pp. 137-156, Apr. 2007.

[4] L. Korowajczuk, LTE, WiMAX and WLAN Network Design, Optimization and Performance Analysis. Wiley, 2011.

[5] M. Shi, Y. Bar-Ness, and W. Su, "Adaptive estimation of the number of transmit antennas," in Proc. IEEE MILCOM, 2007, pp. 1-5.

[6] O. Somekh, O. Simeone, Y. Bar-Ness, and W. Su, "Detecting the number of transmit unauthorized or cognitive receivers in MIMO systems," in Proc. IEEE MILCOM, 2007, pp. 1-5.

[7] V. Choqueuse, S. Azou, K. Yao, and G. Burel, "Blind modulation recognition for MIMO systems," J. Military Technical Academy Review, vol. XIX, pp. 183-196, Jun. 2009.

[8] K. Hassan, I. Dayoub, W. Hamouda, C. Nzeza, and M. Berbineau, "Blind digital modulation identification for spatially-correlated MIMO systems," IEEE Trans. Wireless Commun., vol. 11, pp. 683-693, Feb. 2012.

[9] M. S. Mühlhaus, M. Öner, O. A. Dobre, H. U. Jkel, and F. K. Jondral, "Automatic modulation classification for MIMO systems using fourthorder cumulants," in Proc. IEEE VTC Fall, 2012, pp. 1-5.

[10] — "A novel algorithm for MIMO signal classification using higherorder cumulants," in Proc. IEEE RWS, 2013, pp. 1-5.

[11] V. Choqueuse, M. Marazin, L. Collin, K. Yao, and G. Burel, "Blind recognition of linear space time block codes: A likelihood-based approach," IEEE Trans. Signal Process., vol. 58, pp. 1290-1299, Mar. 2010.

[12] V. Choqueuse, K. Yao, L. Collin, and G. Burel, "Hierarchical spacetime block code recognition using correlation matrices," IEEE Trans. Wireless Commun., vol. 7, pp. 3526-3534, Sep. 2008

[13] — "Blind recognition of linear space time block codes," in Proc. IEEE ICASSP, 2008, pp. 2833-2836.

[14] Y. A. Eldemerdash, M. Marey, O. A. Dobre, G. K. Karagiannidis, and R. Inkol, "Fourth-order statistics for blind classification of spatial multiplexing and Alamouti space-time block code signals," accepted in IEEE Trans. Commun., Mar. 2013.

[15] M. DeYoung, R. Heath, and B. Evans, "Using higher order cyclostationarity to identify space-time block codes," in Proc. IEEE GLOBECOM, 2008, pp. 1-5.

[16] M. Shi, Y. Bar-Ness, and W. Su, "STC and BLAST MIMO modulation recognition," in Proc. IEEE GLOBECOM, 2007, pp. 3034-3039.

[17] M. Marey, O. A. Dobre, and R. Inkol, "Classification of space-time block codes based on second-order cyclostationarity with transmission impairments," IEEE Trans. Wireless Commun., vol. 11, pp. 2574-2584, Jul. 2012.

[18] E. Larsson and P. Stoica, Space-Time Block Coding for Wireless Communication. Cambridge Press, 2003.

[19] V. Tarokh, H. Jafarkhani, and A. Calderbank, "Space time block codes from orthogonal designs," IEEE Trans. Inform. Theory, vol. 45, pp. 744 765, Jul. 1999.

[20] A. Papoulis and S. Pillai, Probability, Random Variables and Stochastic Processes. McGraw-Hill, 2001.

[21] N. Beaulieu and C. Cheng, "Efficient Nakagami-m fading channel simulation," IEEE Trans. Veh. Technol., vol. 54, pp. 413-424, Mar. 2005.

[22] M. E. Johnson, Multivariate Statistical Simulation. Wiley, 1987.

[23] D. Watkins, Fundamentals of Matrix Computations. Wiley, 2002. 